\title{
Oxygen uptake kinetics during low intensity exercise: relevance for rate adaptive pacemaker programming
}

Thorsten Lewalter, Hans Rickli, Dean MacCarter, Werner Jung, Rainer Schimpf, Peter Schwartze, Reto Candinas, Berndt Lüderitz

\begin{abstract}
Objective-To establish a normal database for oxygen uptake $\left(\dot{V}_{2}\right)$ kinetics during low intensity treadmill exercise (LITE) testing, to be used as a guideline for programming rate adaptive pacemakers, and to determine its relation to $\dot{\mathrm{V}} \mathrm{O}_{2}$ at anaerobic threshold and peak exercise.

Design- $\dot{V}_{2}$ kinetics during LITE were compared with $\dot{\mathrm{V}} \mathrm{O}_{2}$ at anaerobic threshold and at peak exercise.
\end{abstract}

Setting-LITE testing is applicable during ambulatory or hospital care and can even be performed by patients with reduced cardiac capacity.

Patients -60 healthy subjects (23 women, 51.6 (SD 20.4) years; 37 men, $42 \cdot 2(16 \cdot 2)$ years).

Interyentions-Treadmill exercise testing with "breath by breath" gas exchange monitoring using the LITE protocol for steady state, submaximal exercise, and the ramping incremental treadmill exercise (RITE) protocol for peak exercise.

Main outcome measures-Mean response time of $\dot{V} o_{2}$, mean oxygen deficit, and $\dot{V} o_{2}$ at anaerobic threshold $\left(\dot{\mathbf{V}} \mathrm{O}_{2}-\mathbf{A T}\right)$ and at peak exercise (Vंo ${ }_{2}$-peak) were determined.

Results-(1) LITE protocol: mean response time of $\dot{\mathrm{Vo}}_{2}=35.1(9.9) \mathrm{s}$; oxygen deficit $=418.3(47.9) \mathrm{ml}$; oxygen deficit/ $/ \mathrm{O}_{2}$ time index $=54 \cdot 7$ (7.4). (2) RITE protocol: $\quad \dot{ } o_{2}-A T=22 \cdot 1 \quad(5 \cdot 7)$ $\mathrm{m} / \mathrm{kg} / \mathrm{min}$; heart rate at anaerobic threshold $=120.1(3.6)$ beats $/ \mathrm{min}$; Vo $\mathbf{o}_{2}-$ peak $=37.6(10.7) \mathrm{ml} / \mathrm{kg} / \mathrm{min}$; peak heart rate $=167.8(19.3)$ beats $/ \mathrm{min}$. The mean response time and oxygen deficit $/ \mathrm{Vo}_{2}$ time index were significantly correlated to $\dot{V} \mathrm{O}_{2}$ peak and $\dot{V} o_{2}-A T(P<0 \cdot 01)$.

Conclusions- $\mathrm{Vo}_{2}$ kinetics calculated in healthy controls may serve as a control database for assessing the rate response programming of pacemakers and its influence on $\dot{V} o_{2}$ during LITE. Because aerobic capacity below the anaerobic threshold is more likely to represent activity in daily life and the kinetics of $\dot{V} o_{2}$ are significantly related to $\dot{V} \mathrm{O}_{2}$ at anaerobic threshold and peak exercise, LITE may provide a clinically useful correlate to peak exercise testing.

(Heart 1997;77:168-172)

Keywords: rate adaptive pacing; oxygen uptake kinetics; submaximal exercise
Oxygen uptake in the lungs begins to increase immediately after the start of muscular exercise. The rate of change or the kinetics of pulmonary oxygen uptake has been studied for the past 50 years to develop a suitable model for the clarification of its mechanism and to determine its relation to physical condition or disease. ${ }^{1-7}$ Although numerous mechanisms have been proposed to model oxygen uptake kinetics, the one offered by Barstow et al appears to be the most acceptable and applicable for the evaluation of normal subjects and abnormal oxygen kinetics in patients with cardiovascular disease. ${ }^{89}$ Barlow's model of oxygen uptake kinetics consists of a three phase model: the first two phases represent the actual dynamic phase of oxygen kinetics and the third phase reflects the attained steady state phase. A majority of the oxygen deficit and the contribution of venous oxygen stores to pulmonary oxygen uptake kinetics are mostly in phase 1 . It has been shown by various investigators that the kinetics of phases 1 and 2 or the time to reach steady state are dependent both on the work rate and on the subject's physical condition. ${ }^{2} 3$ Slower oxygen uptake kinetics have been shown to occur in various disease states; however, limited studies have been conducted to quantitate any difference with rate adaptive pacing and to determine the reliability of measurements of oxygen kinetics. ${ }^{10}{ }^{11}$ In addition, little has been published to establish a control database to serve as a guideline for programming rate adaptive pacemaker devices and to describe the correlation of oxygen uptake kinetics with submaximal or maximum exercise performance in healthy subjects.

\section{Methods}

\section{STUDY GROUP}

The study group consisted of 60 healthy subjects with a mean age of $45 \cdot 8$ (SD 18.3) years (37 men: $42 \cdot 2(16 \cdot 2)$ years, body surface area $195.0(13.19) \mathrm{cm}^{2} ; 23$ women: $51.6(20.4)$ years, body surface area $165.9(12 \cdot 1) \mathrm{cm}^{2}$ ) who were tested using both peak and low intensity exercise. Informed consent was obtained from all subjects. Before exercise testing, a physical examination, patient history, electrocardiogram at rest, echocardiography, routine laboratory tests, and spirometry were examined: none showed any abnormal physical condition or disease state. None of the subjects was receiving chronic drug treatment.

\section{EXERCISE TESTING}

The stress tests were performed on a treadmill

Strasse 25, 53105 Boud-

Accepted for publication 12 November 1996 
with the collection of breath by breath gas exchange on a CPX/D system from Medical Graphics Corporation (St Paul, Minneapolis, USA), using a rapidly responding zirconia fue cell for the oxygen analyser and an infrared carbon dioxide analyser, in conjunction with a disposable pneumotachograph for flow measurements. The expired gas was collected in real time using a gas drying sampling circuit and a low rebreathing dead space pneumotachograph of about $20 \mathrm{ml}$, then analysed using a waveform analyser with automatic phase alignment of flow with the oxygen and carbon dioxide signals. The minute ventilation data were stored for analysis and playback using an eight breath moving average sampling technique. The minute ventilation signal was determined at body temperature and pressure saturated units. During exercise, the electrocardiogram and heart rate were continuously monitored with a CASE 15 monitor (Marquette Corporation, Milwaukee, Wisconsin, USA). Two protocols representing peak and low intensity exercise were used.

Ramping incremental treadmill exercise (RITE) This protocol was used to determine peak exercise, which has been described in detail

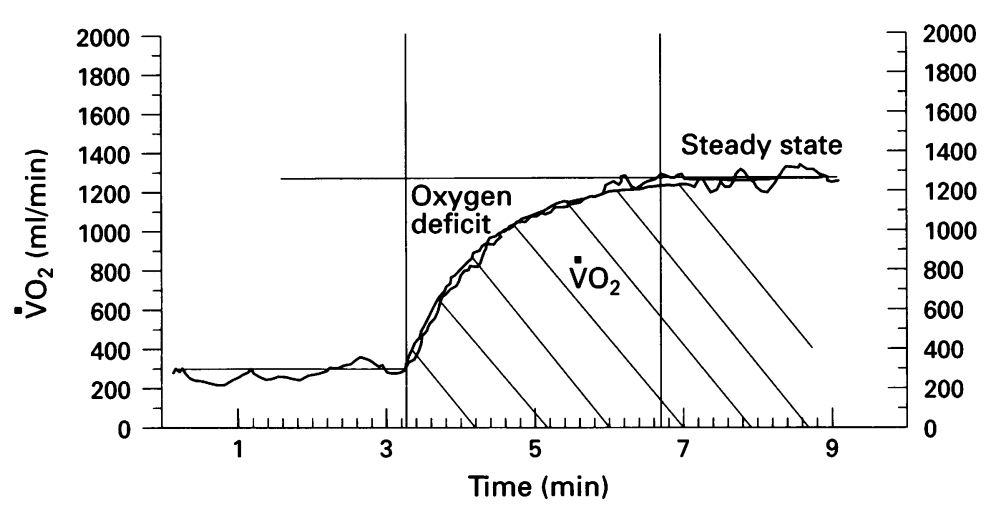

Figure 1 The principles of oxygen deficit and oxygen uptake $\left(\mathrm{VO}_{2}\right)$ kinetics determination. The oxygen defict is the amount of oxygen which has not been taken up or provided beyond the true oxygen uptake from rest to steady state for a specific workload, as indicated by the oxygen uptake at steady state. An exponential oxygen uptake regression curve (exp fit) was used for calculating oxygen deficit and oxygen uptake.

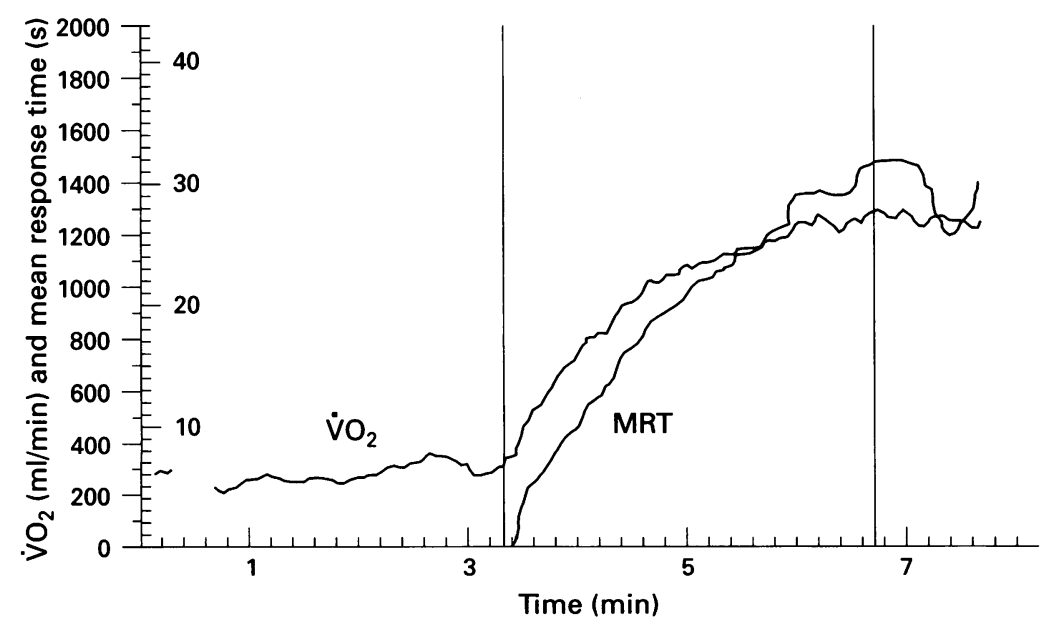

Figure 2 Use of the mean response time (MRT) to determine the onset of steady state. The left marker tags the onset of exercise with the oxygen uptake $\left(\mathrm{VO}_{2}\right)$ steadily increasing. The software calculates the mean response time at each breath using the Whipp formula. As compared with oxygen uptake, the mean response time more clearly indicates the onset of steady state, which is an important point for subsequent calculations. previously. ${ }^{12}$ Heart rate, oxygen uptake and minute ventilation at rest, anaerobic threshold, and peak exercise were measured. Linear regression analysis was used to analyse the relation between heart rate and minute ventilation, oxygen uptake, and work rate both below and above the anaerobic threshold for each patient. The anaerobic threshold was detected in accordance with the $\mathrm{V}$ slope method reported by Beaver and Wasserman. ${ }^{13}$

\section{Low intensity treadmill exercise (LITE)}

This protocol at a $35 \mathrm{~W}$ constant external workload level was used to simulate daily slow walking activity. ${ }^{14}$ After two minutes at rest, the patient was exercised for six minutes on a treadmill with a workload of $35 \mathrm{~W}$, followed by a recovery phase of three minutes. For the determination of resting oxygen uptake, heart rate, and minute ventilation values, only the last 30 seconds of rest before the onset of exercise were averaged, in order to include only true resting steady state data. Subjects were instructed how to walk on the treadmill at their calculated speed (according to body weight) and $8 \%$ treadmill grade to familiarise themselves with the test. All began exercise after the treadmill had reached its programmed speed and grade and the computed $35 \mathrm{~W}$ external workload was confirmed on the monitor, in order to eliminate the mechanical delay in the treadmill and to subject the controls to an instantaneous workload. Therefore any error in the heart rate and minute ventilation kinetic measurements was minimised. The onset of exercise was marked in the computer when the subjects placed their second foot down on the treadmill, or took their first full step. Heart rate, oxygen uptake, and minute ventilation were determined at rest and at the steady state level, which was attained about two to three minutes after the onset of exercise.

\section{OXYGEN UPTAKE KINETICS MEASUREMENT}

As reported by Whipp, ${ }^{2}$ the calculation formula for the oxygen deficit-comprised of oxygen extraction and the change in venous oxygen stores-is as follows:

$$
\text { Oxygen deficit }=\Delta \dot{\mathrm{VO}}_{2} / k
$$

whereby $\Delta \dot{\mathrm{VO}}_{2}$ equals the difference between the resting $\dot{\mathrm{VO}}_{2}$ and steady state (STS) $\dot{\mathrm{VO}}_{2}$ and $k$ is the time or rate constant of the rise in $\dot{\mathrm{VO}}_{2}$ with respect to time (see also fig 1). The mean response time, or the inverse of the rate constant for oxygen uptake kinetics, is then calculated by the following equation:

Mean response time $=$ oxygen deficit $/ \Delta \dot{\mathrm{VO}}_{2}$ The Whipp formula can be expanded by:

$\mathrm{t}$ (time from rest to STS) $\times \Delta \dot{\mathrm{VO}}_{2}-$ cumulative $\dot{\mathrm{VO}}_{2}$ (rest to STS) = oxygen deficit,

to the resulting formula:

Mean response time $=(1 / k)=$ [t (rest to STS) $\times \Delta \dot{\mathrm{VO}}_{2}-$ cumulative $\dot{\mathrm{VO}}_{2}$ (rest to STS)] $/ \Delta \dot{\mathrm{VO}}_{2}$ 
Table 1 Heart rate (HR), work rate, and oxygen uptake $\left(\dot{V}_{2}\right)$ at anaerobic threshold $(A T)$ and at peak exercise

\begin{tabular}{lllllll}
\hline & $\begin{array}{l}\text { HR-AT } \\
\text { (beats/min) }\end{array}$ & $\begin{array}{l}\text { Work load } A T \\
\text { (W) }\end{array}$ & $\begin{array}{l}\dot{V} \mathrm{O}_{2}-A T \\
(\mathrm{ml} / \mathrm{kg} / \mathrm{min})\end{array}$ & $\begin{array}{l}\text { HR-peak } \\
(\text { (bpm) }\end{array}$ & $\begin{array}{l}\text { Work load peak } \\
\text { (watts) }\end{array}$ & $\begin{array}{l}\dot{V} \mathrm{O}_{2}-\text { peak } \\
(\mathrm{ml} / \mathrm{kg} / \mathrm{min})\end{array}$ \\
\hline Study group (n=60) & $120 \cdot 1(13 \cdot 6)$ & $86 \cdot 7(37 \cdot 8)$ & $22 \cdot 1(5 \cdot 7)$ & $167 \cdot 8(19 \cdot 3)$ & $218 \cdot 6(87 \cdot 7)$ & $37 \cdot 6(10 \cdot 7)$ \\
Women (n=23) & $124 \cdot 3(14 \cdot 4)$ & $60 \cdot 9(20 \cdot 6)$ & $19 \cdot 1(3 \cdot 6)$ & $162 \cdot 1(21 \cdot 3)$ & $145 \cdot 5(42 \cdot 5)$ & $30 \cdot 0(6 \cdot 6)$ \\
Men (n=37) & $117 \cdot 5(12 \cdot 6)$ & $103 \cdot 7(34 \cdot 9)$ & $24 \cdot 0(5 \cdot 9)$ & $171 \cdot 3(17 \cdot 3)$ & $267 \cdot 0(70 \cdot 4)$ & $42 \cdot 3(10 \cdot 1)$ \\
\hline
\end{tabular}

The $\mathrm{VO}_{2}$ data are filtered using a default filter size of 9. The filter was implemented so that there was no phase lag between the raw data and the filtered data. In the next step, the software calculated the mean response time at every breath after the onset of exercise using the Whipp formula, while assuming that steady state was achieved at a given breath. To reduce the extremely high sensitivity of the mean response time, it was filtered using the same median filter used to filter the $\dot{V} \mathrm{O}_{2}$ data. Guided by the filtered mean response time data, which clearly indicates the steady state, the user can adjust the computer screen cursor to indicate the point of onset of the steady state. The screen is continuously updated every time the cursor is moved and displays the following:

- $r$ value or correlation coefficient of the current exponential fit depending on the position of the right cursor

- $\dot{\mathrm{VO}}_{2}$ at steady state indicated by the marker on the display

- cumulative $\dot{\mathrm{VO}}_{2}$ from rest to steady state

- oxygen deficit

- mean response time

- time in seconds from rest to the selected onset of steady state.

All tests were analysed using this software. Special attention was given to the determination of the steady state by using the mean response time and the oxygen uptake curve profile (fig 2).

In addition, a new parameter called the "oxygen deficit $/ \mathrm{VO}_{2}$ time index", directly correlating the oxygen deficit to the cumulative $\dot{\mathrm{VO}}_{2}$ from rest to steady state, was calculated from the following formula:

Oxygen deficit $/ \mathrm{VO}_{2}$ time index $=$ oxygen deficit/cumulative $\dot{\mathrm{VO}}_{2}$ (rest to STS) $\times$ t (rest to STS)

\section{STATISTICAL METHODS}

All results are expressed as the mean (SD). Group mean differences were assessed using a paired or unpaired Student's $t$ test. A P value of $<0.05$ was considered statistically significant. Univariate and stepwise non-prioritised correlation regression analysis were performed among several variables.

Table 2 Oxygen deficit, mean response time (MRT) of oxygen uptake $\left(\dot{V}_{2}\right)$, and the oxygen deficit/ $/ \mathrm{O}_{2}$ time index for the entire study group

\begin{tabular}{llll}
\hline & $\begin{array}{l}\text { Oxygen deficit } \\
(\text { mllkg) }\end{array}$ & $\begin{array}{l}M R T \\
(s)\end{array}$ & $\begin{array}{l}\text { Oxygen deficit/ } \dot{V O}_{2} \\
\text { time index }\end{array}$ \\
\hline Study group (n=60) & $5 \cdot 9(2 \cdot 1)$ & $35 \cdot 1(9 \cdot 9)$ & $54 \cdot 7(17 \cdot 4)$ \\
Women (n= 23) & $7 \cdot 5(2 \cdot 4)$ & $38 \cdot 2(10 \cdot 4)$ & $59 \cdot 9(18 \cdot 1)$ \\
Men $(\mathrm{n}=37)$ & $5 \cdot 5(1 \cdot 9)$ & $33 \cdot 2(9 \cdot 2)$ & $51 \cdot 4(16.4)$ \\
\hline
\end{tabular}

\section{Results}

\section{PEAK EXERCISE TESTING}

Using the full effort symptom limited RITE protocol, the cardiopulmonary response to both low and high exercise intensities was evaluated and is shown in table 1 with a $\mathrm{Vo}_{2}$ at anaerobic threshold of $22 \cdot 1(5 \cdot 7) \mathrm{ml} / \mathrm{kg} / \mathrm{min}$ and at peak exercise $37.6(10.7) \mathrm{ml} / \mathrm{kg} / \mathrm{min}$. For the entire study group, the heart rate to work rate slope from rest to peak exercise was $0.47(0.18)$ beats $/ \mathrm{min} / \mathrm{watt}$, with a mean correlation coefficient of $r=0.96$, indicating a mean increase of approximately 5 beats per increase of 10 watts of external treadmill work performed. As already reported ${ }^{14}$ women generated a significantly steeper slope throughout exercise than men: $0.57(0.18) v 0.41(0.15)$; $P<0.001$.

LOW INTENSITY EXERCISE TESTING

Using the LITE protocol to characterise the sinus node response during the early, dynamic phase of exercise, the following variables were determined. Heart rate at rest was 80 (13) beats/min compared with 105 (18) beats/min at the steady state level. At resting and steady state levels, minute ventilation was 10.9 (3.2) and $25 \cdot 2(4.9) \mathrm{l} / \mathrm{min}$ respectively. Resting oxygen uptake was $4 \cdot 1 \quad(1 \cdot 3) \mathrm{ml} / \mathrm{kg} / \mathrm{min}(1 \cdot 2$ METS) compared with an average oxygen uptake at steady state of $9 \cdot 7(3.8) \mathrm{ml} / \mathrm{kg} / \mathrm{min}$ (2.8 METS)-thus there was a substantial increase of $136 \%$ with a low intensity treadmill work. The results of the oxygen uptake kinetics are shown in table 2 . The oxygen deficit for the entire study group was $418 \mathrm{ml}$ or $5.9 \mathrm{ml} / \mathrm{kg}$, with a significant difference between men and women $(P<0.001)$, whereas the mean response time and the oxygen deficit $/ \dot{\mathrm{V}}_{2}$ time index showed only a slight non-significant difference between the genders $(P=0.056$ and $P=0.066$ respectively).

\section{CORRELATION BETWEEN LOW AND PEAK}

EXERCISE TESTING

The results from the univariate correlation analysis between oxygen uptake kinetics and peak exercise data are shown in table 3. All submaximal oxygen uptake indices were significantly correlated with age, heart rate, and oxygen uptake at peak exercise, whereas no correlation with body surface area could be detected. The mean response time and the oxygen deficit $/ \mathrm{VO}_{2}$ time index were significantly correlated with oxygen uptake at anaerobic threshold, whereas the oxygen deficit was correlated with the heart rate to work rate relation from rest to peak exercise. In its correlation with oxygen uptake at anaerobic threshold and peak exercise, the oxygen deficit $/ \mathrm{VO}_{2}$ time index generated the highest correlation coefficients among the variables studied. Per- 
Table 3 Univariate correlation analysis

\begin{tabular}{|c|c|c|c|c|c|c|}
\hline & $\begin{array}{l}\text { Age } \\
\text { (years) }\end{array}$ & $\begin{array}{l}\text { Body surface area } \\
\left(\mathrm{cm}^{2}\right)\end{array}$ & $\begin{array}{l}\dot{V} \mathrm{O}_{2}-A T \\
(\mathrm{ml} / \mathrm{kg} / \mathrm{min})\end{array}$ & 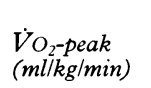 & $\begin{array}{l}\text { Heart rate peak } \\
\text { (beats/min) }\end{array}$ & $\begin{array}{l}H R / W R \\
\text { (rest-peak) } \\
\text { (beats/watt) }\end{array}$ \\
\hline MRT(s) & $\begin{array}{l}P<0.0001^{\star} \\
R=0.48\end{array}$ & $\begin{array}{l}P=0.84 \\
R=-0.03\end{array}$ & $\begin{array}{l}P=0.01^{\star} \\
\mathrm{P}=-0.33\end{array}$ & $P=0.0003^{\star}$ & $\begin{array}{l}\mathrm{P}=0.0002^{\star} \\
\mathrm{P}=-0.46\end{array}$ & $\begin{array}{l}P=0.17 \\
R=-0.18\end{array}$ \\
\hline Oxygen deficit & $\begin{array}{l}R=0.48 \\
P=0.0032^{\star}\end{array}$ & $\begin{array}{l}R=-0.03 \\
P=0.7\end{array}$ & $\begin{array}{l}R=-0.33 \\
P=0.13\end{array}$ & $P=0.006^{\star}$ & $\mathrm{P}=0.006^{\star}$ & $\begin{array}{l}R=-0.18 \\
P=0.01^{\star}\end{array}$ \\
\hline & $\mathrm{R}=0.38$ & $R=-0.05$ & $\mathrm{R}=-0 \cdot 2$ & $\mathrm{R}=-0.35$ & $\mathrm{R}=-0.35$ & $\mathrm{R}=-0.32$ \\
\hline Qxygen deficit/ & $P=0.0002^{\star}$ & $P=0.8$ & $P=0.003^{\star}$ & $P=0.0002^{\star}$ & $P<0.0004^{\star}$ & $P=0.28$ \\
\hline $\mathrm{VO}_{2}$ time index & $\mathrm{R}=0.46$ & $R=-0.03$ & $R=-0.38$ & $R=-0.46$ & $R=-0.44$ & $R=-0 \cdot 14$ \\
\hline
\end{tabular}

AT, anaerobic threshold; $\dot{\mathrm{VO}}_{2}$, oxygen uptake; WR, work rate: MRT, mean response time.

$\star \mathrm{P}<0 \cdot 05$.

forming a non-prioritised stepwise regression correlation analysis, it was shown that peak oxygen uptake was the only independent determinant of the mean response time $(\mathrm{F}=$ $21 \cdot 5)$ and the oxygen deficit $/ \mathrm{VO}_{2}$ time index $(\mathrm{F}$ $=18 \cdot 6$ ), whereas the oxygen deficit was independently influenced only by age $(F=4 \cdot 7)$.

\section{Discussion}

Over the past 10 years, emphasis in the field of rate adaptive pacing has focused mainly on the influence of rate modulation on peak exercise capacity, with a shift in interest during recent years towards the mid-point of exercise or anaerobic threshold. Among others, Hayes and coworkers evaluated protocols and parameters to assess the influence of rate adaptation during subanaerobic threshold daily life activities, as represented by the slow and brisk walk concept. ${ }^{15}$ Heart rate modulation, however, is only one among other variables such as stroke volume, blood pressure, or ventilation which change abruptly at the onset of submaximal exercise. It is still a controversial issue-espe- cially in patients with impaired left ventricular function-as to what should be the pacemaker's desired speed of response, paced rate slope, or target paced rate at a given workload level under submaximal conditions. Attempts have been made to relate sinus node activity during exercise to changes in ventilation or workload as a guideline for the programming of rate adaptive devices. ${ }^{141617}$ Others have investigated the impact of rate response on functional capacity during psychological testing using perceived exertion scales and quality of life questionnaires as a subjective indicator of the appropriateness of the cardiopulmonary response to exercise. ${ }^{18}{ }^{19}$ Performing peak exercise testing in patients with rate adaptive pacemakers, the peak workload achieved-in combination with oxygen uptake or exercise duration-is a clear indicator of functional capacity. However, it is the philosophy of light exercise subanaerobic threshold protocols, like the LITE protocol or the slow/brisk walk concept, that most patients could perform the tests for long periods, since the workload is intentionally selected to be below the anaerobic
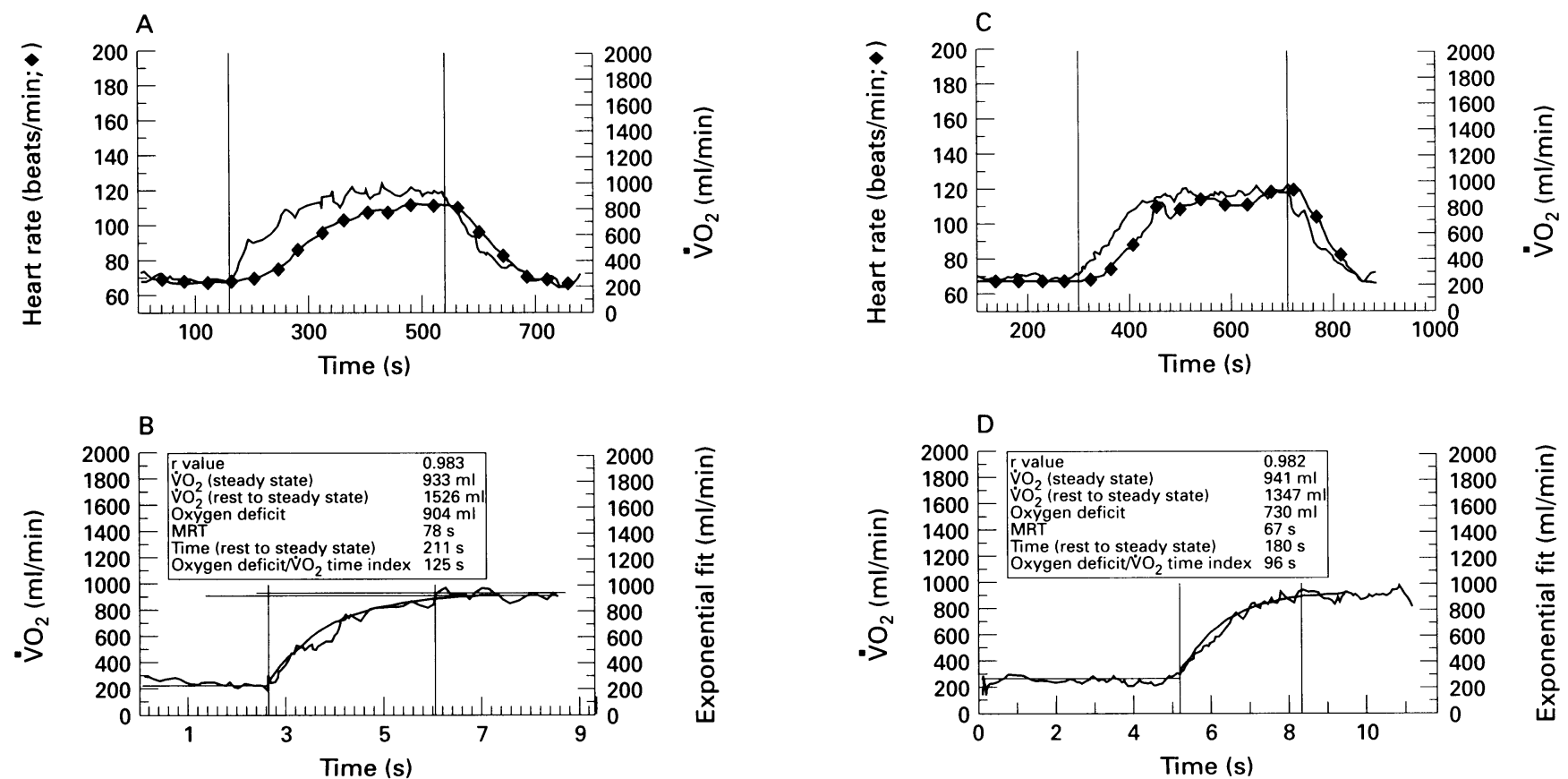

Figure 3 Potential clinical application of oxygen uptake kinetics in the field of rate adaptive pacing. (A) A LITE protocol exercise test performed in a patient with a VVIR minute ventilation based, rate adaptive pacemaker. The test was conducted with the pacemaker's speed of response programmed to the nominal value of medium. Heart rate response to the constant workload has a significant delay, with a prolonged time of 211 s to reach the indicated heart rate at steady state level of about 110 beats/min. The corresponding oxygen uptake and calculated values for oxygen uptake kinetics are shown in panel (B). Because the rate response was obviously too slow, with reduced oxygen uptake kinetics compared to the control database, the pacemaker's speed of response was enhanced by programming it to fast $(C)$. The paced rate now reaches the steady state level in $180 \mathrm{~s}$ after the onset of exercise, indicating an improved speed of response compared to the previous test $(211 \mathrm{~s})$. Evaluation of oxygen uptake (VO, panel (D)) shows a reduced and therefore improved oxygen deficit of $730 \mathrm{ml} v 904 \mathrm{ml}$, or a reduction of $19.2 \%$. The mean response time (MRT) shortened from 78 to $67 \mathrm{~s}(14 \cdot 1 \%)$ with the

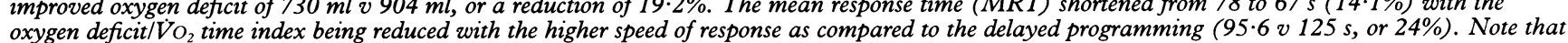
the oxygen uptake level attained during steady state showed only a slight difference (941-933 ml). 
threshold. Physicians began to focus away from previously approved endpoints in exercise testing, such as peak workload or exercise duration, in order to differentiate between a "good" or "poor" cardiopulmonary response. 11220 To generate an adequate oxygen uptake relative to the body's metabolic demand is the goal of the cardiopulmonary response to exercise. Therefore it appears logical that would be useful to measure oxygen uptake kinetics at the onset of low intensity, daily type exercise. It has been shown that the mean response time or the time constant for oxygen uptake can be used as a sensitive and discriminatory parameter reflecting impaired cardiac reserve in patients with reduced left ventricular function. ${ }^{7}$ Moreover the paced rate at the onset of exercise has been shown to have a significant influence on the amount of the oxygen deficit, hence its importance for appropriate programming in rate adaptive pacemaker patients ${ }^{11}$ (see also fig 3). However, there are no data that serve as a control database describing oxygen uptake kinetics in healthy subjects of a large sample size and also as a guide for assessing the influence of rate adaptive pacing on submaximal aerobic capacity. The data presented in this study show that, in contrast to the oxygen deficit which was independently influenced only by age, the mean response time and the oxygen deficit $/ \mathrm{VO}_{2}$ time index were significantly correlated with oxygen uptake at the anaerobic threshold and peak exercise, with peak oxygen uptake being the only independent determinant. Of these two variables, the oxygen deficit $/ \mathrm{VO}_{2}$ time index generated the higher correlation coefficient describing its relation to oxygen uptake, thus indicating that this variable may be a more significant reflection of low intensity exercise capacity relative to peak exercise.

A critical point in the past was the method and reliability of the available software, especially with regard to determining the onset of the steady state phase. By the use of a newly implemented, filtered "moving" mean response time calculation, which clearly displays the dynamic and the steady state phase, it is now much easier to perform reproducible oxygen uptake kinetics measurements, even for inexperienced users.

\section{STUDY LIMITATIONS}

Whether the normal oxygen kinetics database can serve as a guide for pacemaker programming in patients with a concomitant impairment of left ventricular function or lung disease remains unclear and will require further evaluation. A critical point with the use of the LITE protocol is the actual procedure. If the patient is not at true rest before the onset of exercise or starts the protocol with a warm up phase, the data obtained are not reliable. Even if "low intensity" sounds like an "easy to do" method, more emphasis should be placed on the details of how to conduct the test.

\section{CLINICAL IMPLICATIONS}

In order to improve the patient's submaximal capacity, compared to that found with a pre- pacemaker, chronotropically insufficient rate, the pacemaker's rate response should be programmed to generate oxygen uptake kinetics that closely simulate normal physiology. Knowing the significantly positive correlation of oxygen uptake kinetics with peak oxygen uptake, one can also expect to improve maximum exercise capacity if, for example, the effect of an appropriately programmed paced rate response is maintained throughout exercise to peak intensities. If this could be demonstrated in future pacemaker studies, low intensity exercise testing could substitute for peak exercise, which would be much more comfortable for the patient and easy to apply for the physician during pacemaker follow up procedures.

This study was partly presented at the 14th annual scientific sessions of the North American Society of Pacing and Electrophysiology, May 5-8, 1993, San Diego, California, USA.

1 Donald KW, Bishop JM, Wade OL. A study of minute-tominute changes of arterio-venous oxygen content difference, oxygen uptake and cardiac output and rate of achievement of a steady-state during exercise in rheumatic heart disease. FClin Invest 1954;33:1146-67.

2 Whipp BJ. Rate constant for the kinetics of oxygen uptake during light exercise. F Appl Physiol 1971;30:261-3.

3 Hagberg JM, Nagle FJ, Carlson JL. Transient oxygen uptake response at the onset of exercise. $\mathcal{F}$ Appl Physio 1978;44:90-2.

4 Sietsema KE, Cooper DM, Perloff JK, Rosove MH, Child JS, Canobbio MM, et al. Dynamics of oxygen uptake during exercise in adults with cyanotic congenital heart disease. Circulation 1986;73:1137-44.

5 Gildein P, Mocellin R, Kaufmehl K. Oxygen uptake transient kinetics during constant-load exercise in children after operations of ventricular septal defect, tetralogy of after operations of ventricular septal defect, tetralogy of
Fallot, transposition of the great arteries, or tricuspid Fallot, transposition of the great arteries,
valve atresia. Am $\mathcal{A}$ Cardiol 1994;74:166-9.

6 Sietsema KE. Oxygen uptake kinetics in response to exercise in patients with pulmonary vascular disease. Am Rev cise in patients with pulmonary
Respir Dis 1992;145:1052-7.

7 Koike A, Hiroe M, Adachi H, Yajima T, Yamauchi Y, Nogami A, et al. Oxygen uptake kinetics are determined by cardiac function at onset of exercise rather than peak exercise in patients with prior myocardial infarction. Circulation 1994;90:2324-32.

8 Barstow TJ, Lamarra N, Whipp BJ. Modulation of muscle and pulmonary oxygen uptakes circulatory dynamics during exercise. F Appl Physiol 1990;68:979-89.

9 Whipp BJ. Dynamics of pulmonary gas exchange. Circulation 1987;76:18-28.

10 Casaburi R, Spitzer S, Wassermann K. Effect of altering heart rate on oxygen uptake at exercise onset. Chest 1989;95:6-12.

11 Dailey SM, Bubien RS, Kay GN. Effect of chronotropic response pattern on oxygen kinetics. PACE 1994;17: response

12 Lewalter T, MacCarter D, Jung W, Bauer T, Schimpf $R$ Manz M, et al. The "Low-Intensity Treadmill Exercise" Manz M, et al. The "Low-Intensity Treadmill Exercise" protocol for the programm.

13 Beaver WL, Wasserman K, Whipp BJ. A new method for detecting the anaerobic threshold by gas exchange. $\mathcal{F} A p p$ Physiol 1986;60:2020-7.

14 Lewalter T, MacCarter D, Jung W, Schimpf R, Manz M, Lüderitz $B$. Heart rate to work rate relation throughou peak exercise in normal subjects as a guideline for rateadaptive pacemaker programming. Am $\mathcal{f}$ Cardiol 1995; 76:812-6.

15 Hayes DL, Feldt L, Higano ST. Standardized informa exercise testing for programming rate adaptive pacemakers. PACE 1991;14:1772-6.

16 Wilkoff BL, Corey J, Blackburn G. A mathematical model of the cardiac chronotropic response to exercise. Electrophysiol 1989;3:176-80.

17 Treese N, MacCarter D, Akbulut O, Coutinho M, Baez M, Liebrich $A$, et al. Ventilation and heart rate response during exercise in normals: relevance for rate variable pacing exercise in normals: relevanc

18 Lau CP, Rushby J, Leigh-Jones M, Poloniecki J, Ingram A Sutton $\mathrm{R}$, et al. Symptomatology and quality of life in putton $\mathrm{R}$, et al. Symptomatology and quality of life in patients with rate-responsive pacemakers: a double-blind, 505-12.

19 Oto MA, Muederrisoglu H, Ozin MB, Korkmaz ME, Karamehmetoglu A, Oram A, et al. Quality of life in patients with rate reponsive pacemakers: a randomized, cross-over study. PACE 1991;14:800-6.

20 Francis GS, Rector TS. Maximal exercise tolerance as a therapeutic end point in heart failure-are we relying on the right measure? Am $\mathcal{F}$ Cardiol 1994;73:304-6. 